\title{
Depressive, anxiety, stress, and insomnia symptoms in patients with psoriasis: a cross-sectional study
}

\author{
Moetaza M. Soliman \\ Department of Pharmacy Practice, Faculty of Pharmacy, Mansoura University, Egypt
}

Adv Dermatol Allergol 2021; XXXVIII (3): 510-519

DOI: https://doi.org/10.5114/ada.2020.98726

\begin{abstract}
Introduction: Assessment of psychological well-being in psoriasis patients is recommended. However, studies evaluating depressive, anxiety, stress, and insomnia symptoms in Arabic patients with psoriasis are lacking.

Aim: To quantify levels of psoriasis-related depressive, anxiety, stress, and insomnia symptoms in Arabic patients with psoriasis.

Material and methods: A cross-sectional survey on patients' demographics, disease characteristics, and psychological measures using the Patient Health Questionnaire-9, Generalized Anxiety Disorder 7-item scale, Depression Anxiety Stress Scale-21 (7-item stress subscale), and Insomnia Severity Index. The prevalence and scores of depressive, anxiety, stress, and insomnia symptoms were calculated. Multivariate linear regression models were developed to assess patients' demographics and disease characteristics affecting the psychological measures.

Results: The analysis included 223 patients. The patients reported mild levels of depressive, anxiety, and insomnia symptoms (median (interquartile range (IQR)) scores: 9 (6-14), 6 (4-11), and 12 (4-18), respectively) and a moderate level of stress symptoms (median (IQR) score: 10 (5-14)). The prevalence of depressive, anxiety, stress, and insomnia symptoms were $47.1 \%, 32.7 \%, 59.6 \%$, and $57 \%$, respectively. Multivariate linear regression analyses revealed that for each unit increase in the impact of psoriasis on daily life, there were 5.7 (95\% confidence intervals (CI): 3.7-7.8), 3.8 (95\% Cl: 1.8-5.7), 5.3 (95\% Cl: 3.1-7.4), and 6.5 (95\% Cl: 3.7-9.4) units increase in depression, anxiety, and stress, and insomnia scores, respectively.

Conclusions: The prevalence of depressive, anxiety, stress, and insomnia symptoms in Arabic patients with psoriasis was high. Clinical interventions, screening for psychiatric comorbidities, and consideration of psychotherapy should be implemented in this patient group.
\end{abstract}

Key words: psoriasis, depression, anxiety, stress, insomnia, cross-sectional.

\section{Introduction}

Psoriasis is a dermatological disorder that is known to have a negative impact on patients' health-related quality of life (QoL) [1]. The physical appearance of the disease leads to patient stigmatization and subsequently, patient isolation and avoidance of social interactions. This altered behavior and the impaired QoL may precipitate psychological disorders such as depression, anxiety, and stress [2]. Psoriasis patients are 1.5 times more likely to develop depressive symptoms compared with controls, and more than $20 \%$ of psoriasis patients may experience depression and anxiety [3].

The relationship between psychological distress and disease severity in psoriasis is bidirectional and complex, with inflammation serving as the common factor [4]. Investigators have found that depression, anxiety, and stress can exacerbate psoriasis severity [5], and studies have revealed that treating psychological distress can lead to improvement in psoriasis severity [6]. In addition, researchers have found that treating psoriasis results in improvement in QoL and less depressive symptoms [7]. Biological therapies improved the disease and also led to less frequent comorbid manifestations of depression and anxiety [8-10].

In Arabic countries, few studies have assessed psychological distress in dermatological disorders. In a dermatology clinic in Riyadh, Saudi Arabia, the frequencies of depression and anxiety in dermatological conditions were $14 \%$ and $29 \%$, respectively [11]. Another study from Saudi Arabia reported that the prevalence of depression, anxiety, and stress were $12.6 \%, 22.1 \%$, and $7.5 \%$, respectively [12]. The prevalence of depressive symptoms among patients with dermatological disorders in Muscat,

\footnotetext{
Address for correspondence: Moetaza M. Soliman, Department of Pharmacy Practice, Faculty of Pharmacy, Mansoura University, ElGomhoria St, Mansoura, ElDakahlia, Egypt, phone: 021142911306, e-mail: moetaza13@yahoo.com Received: 25.06.2020, accepted: 5.08.2020. 
Oman, was 24\% [13] and among dermatology clinic patients in Jeddah, Saudi Arabia, was 15.8\% [14]. However, these studies were not specific to psoriasis and did not evaluate stress or insomnia levels and were limited to one clinical centre in an Arabic country.

\section{Aim}

This study aimed to quantify the levels and prevalence of depressive, anxiety, stress, and insomnia symptoms and explore patients' demographics and disease characteristics associated with the psychological measures in Arabic patients with psoriasis.

\section{Material and methods}

\section{Study design}

Patients' data were collected using a cross-sectional study design. An online survey was created and administered to Arabic patients with psoriasis through websites of associations of Arabic patients with psoriasis and social media. The survey informed the patients about the study and obtained patients' consent for participation. Data on patients' socio-demographics (age, gender, level of education, marital status, and country of birth) and disease characteristics (disease onset and disease severity assessed using the self-assessed Simplified Psoriasis Index-severity (sa-SPI-s)) [15] were collected. The impact of psoriasis on daily life was assessed using the sa-SPIpsychosocial impact (sa-SPI-p) [15]. The survey included collecting the main outcome measures that assessed depressive, anxiety, stress, and insomnia symptoms. The survey was created in the Arabic language and validated Arabic versions of all included questionnaires were used. Arabic patients, who were diagnosed with psoriasis, were $\geq 18$ years old, and provided consent were included in the study.

Ethical approval was obtained from the Institutional Ethics Committee prior to the commencement of data collection. The study was conducted in accordance with the Helsinki Declaration of 1975, as revised in 2000.

\section{Sample size}

The sample size for the study was calculated using the formula: $n=Z^{2} P Q / d^{2}[16]$, where $\mathrm{n}$ is the required number of patients, $Z$ is the $Z$ statistics for a level of confidence according to the probability tables ( $Z$ equals 1.96 for $95 \%$ confidence and 1.645 for $90 \%$ confidence), $P$ is the expected prevalence based on previous studies, $Q$ is $100-P$, and $d$ is the precision (5-20\%). According to a meta-analysis on depressive symptoms in patients with psoriasis using patient questionnaires, the prevalence of depressive symptoms was $28 \%$ [3]. The precision is determined based on the value of prevalence (P) [17]. Hence, the sample size for a prevalence of $28 \%$, precision of $6 \%$, and confidence level of $95 \%$ was calculated as 216 patients.

\section{Outcome measures}

\section{Patient Health Questionnaire-9 (PHQ-9)}

The PHQ-9, a scale for assessing depression severity [18], comprises nine questions. Each question is scored from 0 to 3, where a patient selects either not at all (0), several days (1), more than half the days (2), or nearly every day (3) in response to each question, generating a score from 0 to 27 . The nine questions cover the following aspects of depression: little interest in activities; feeling down; little energy; feeling bad about self; and over or under expression of sleep, appetite, and moving/ speaking. The ninth question is regarding thoughts on preference of being dead or intention to self-harm. The PHQ-9 contains an additional question (tenth) on difficulty in performing daily life duties due to depressive symptoms with response options of not difficult at all, somewhat difficult, very difficult, or extremely difficult.

Cut points of $5,10,15$, and 20 were used to generate categorical description of depression severity, where the score was interpreted as the patient is having no or minimal (0-4), mild (5-9), moderate (10-14), moderately severe (15-19), or severe depression (20-27). Because a score of $\geq 10$ indicated that pharmacotherapy should be considered, it was used as the cut point for calculating the prevalence of depression. A score of 2 or 3 in the ninth question was interpreted as presence of suicidal ideation. The Arabic version of the PHQ-9 was developed and validated in 2017 [19].

\section{Generalized Anxiety Disorder 7-item (GAD-7)}

The GAD-7 scale assesses the generalized anxiety disorder [20]. It comprises seven items that measure feeling nervous, anxious, worried too much, and afraid as if something awful might happen, being restless, easily annoyed, and unable to stop worrying. An additional question (eighth) is used to describe how difficult anxiety problems made the daily life duties of the patient. The response to each GAD-7 question was scored from 0 to 3 , similar to that of the PHQ-9, generating a total score of $0-21$. Cut points of 5, 10, and 15 were used to generate categorical description of anxiety severity, where the score was interpreted as the patient is having no or minimal (0-4), mild (5-9), moderate (10-14), or severe (15-21) anxiety. Because a score of $\geq 10$ indicated that further evaluation should be considered, it was used as the cut point for calculating the prevalence of anxiety. Although the GAD-7 scale was developed to assess generalized anxiety disorder, it is sensitive to screening of panic, social anxiety, and post-traumatic stress disorders. The Arabic version of the GAD-7 scale was generated and validated in 2017 [19]. 


\section{7-item stress subscale of the Depression Anxiety} Stress Scale-21 (DASS-21)

The stress subscale of the DASS-21 quantitatively measures stress [21]. It comprises seven statements regarding being touchy, agitated, over-reacting to situations, intolerant to any hindrance, and finding it hard to wind down and difficult to relax. The patients indicated how much each statement applied to them over the past week on a 0-3 scale (0: never, 1: sometimes, 2: often, and 3: almost always), generating a total score of $0-21$. The score interpretation was categorized as normal (0-7), mild (8-9), moderate (10-12), severe (13-16), and extremely severe (17-21) stress. A cut-off score of $\geq 8$ was used for calculating the prevalence of stress [22]. A validated Arabic version of the DASS21 was available [23].

\section{Insomnia Severity Index (ISI)}

The ISI is a self-report scale of patient's perception of insomnia symptoms [24]. It comprises seven items that measure problems associated with sleep over the last month, such as difficulty to initiate sleep, difficulty to stay asleep, waking up really early, worry about having sleep problems, satisfaction/dis-satisfaction with sleep pattern, and interference of sleep problems with daily life activities and how noticeable it is to others. The patients responded to each question on a $0-4$ scale, from less to more severe, generating a score of $0-28$. The score was interpreted as the patient is having no clinically significant insomnia (0-7), mild insomnia (8-14), moderate insomnia (15-21), or severe insomnia (22-28). Because a cut-off score of $\geq 10$ is optimal for detecting insomnia in a community sample [25], it was used as the cut point for calculating the prevalence of insomnia. A validated Arabic version of the ISI was available [26].

\section{Statistical analysis}

Descriptive statistics of all variables are presented. Normally distributed variables are presented as means (SD) and non-normally distributed variables as medians (interquartile range (IQR)). Discrete variables are presented as percentages. Depression, anxiety, stress, and insomnia scores were categorized into dichotomous responses (yes/no) for calculating the prevalence. The Wilcoxon signed-rank test and Kruskal-Wallis test by ranks were used to test differences in outcome measures between different patient sub-groups. Multivariate linear regression analyses were performed to evaluate factors associated with depressive, anxiety, stress, and insomnia symptoms as measured by the PHQ-9, GAD-7 scale, stress subscale of the DASS-21, and ISI, respectively. The variables included in the analyses were age, gender, level of education, psoriasis duration, psoriasis severity, and impact of psoriasis on daily life. Results of the regression analyses are presented as $\beta$ coefficients and $95 \%$ confidence intervals $(\mathrm{Cl})$. A $p$-value of $<0.05$ was considered significant. Stata 10.1 software (Stata Corp., College Station, TX, USA) was used to perform all statistical analyses.

\section{Results}

\section{Patients' characteristics}

A total of 234 patients responded to the survey, of which 11 were excluded due to missing values. The final analysis included 223 patients. Patients' sociodemographics and clinical characteristics in total and stratified by gender are presented in Table 1 . The mean \pm SD age of the patients was $34.8 \pm 10.6$ years and 126 (56.5\%) patients were men. The median (IQR) psoriasis duration was 12 (5-20) years. The psoriasis severity as measured by the sa-SPI-s was mild (median (IQR): 7.3 (3-16)). The median (IQR) impact of psoriasis on daily life as measured using the sa-SPI-p was 5.5 (3-9). Sixty percent of the patients had a university degree and $58.7 \%$ were married. Patients belonged to 11 Arabic countries. Women had a longer disease duration $(p=0.04)$, were more educated than men $(p<0.0001)$, and majority of them were single $(p=0.003)$.

\section{Depressive symptoms}

The median (IQR) PHQ-9 score of the patients was 9 (6-14), reflecting mild depressive symptoms. Approximately $23.8 \%, 14.8 \%$, and $8.5 \%$ of the patients showed moderate, moderately severe, and severe depressive symptoms, respectively (Figure 1). The prevalence of depressive symptoms was $47.1 \%$ (95\% Cl: 40.5-54.0\%; Figure 2). In response to the nine questions of the $\mathrm{PHQ}-9$, $83 \%$ of the patients reported feeling down, depressed, or hopeless and $33.6 \%$ reported thoughts of being better off dead. The prevalence of suicidal ideation was $18.4 \%$ (95\% Cl: 13.3-23.5\%). In response to the tenth question on how depressive symptoms affected work and social life, 70 (32.4\%), 119 (55.1\%), 21 (9.7\%), and 6 (2.8\%) patients reported not difficult at all, somewhat difficult, very difficult, and extremely difficult, respectively.

Women reported higher levels of depressive symptoms than men, with a median (IQR) of 11 (7-16) and 9 (6-12), respectively ( $p=0.02$; Table 2$)$. Patients with severe psoriasis showed the highest median (IQR) PHQ-9 score (13 [9-16]) compared with those with moderate $(9$ [6-14]) and mild (8 [5-12]) disease $(p=0.0004)$. Patients with a higher impact of psoriasis on their daily life showed higher levels of depressive symptoms ( $p=0.0001)$. Multivariate linear regression analysis for factors associated with depression score revealed that for each unit increase in the impact of psoriasis on daily life, there was 2.8 (95\% Cl: 0.8-4.9) units increase in the PHQ-9 score for sa-SPIp scores ranging from 4 to 7 and $5.7(95 \% \mathrm{Cl}$ : 3.6-7.8) units increase for sa-SPI-p scores > 7 (Table 3). 
Table 1. Patients' socio-demographics and disease characteristics

\begin{tabular}{|c|c|c|c|c|c|c|c|}
\hline \multirow[t]{2}{*}{ Patients' characteristics } & \multicolumn{2}{|c|}{ Total } & \multicolumn{2}{|c|}{ Female } & \multicolumn{2}{|c|}{ Male } & \multirow[t]{2}{*}{$P$-value } \\
\hline & $n$ & (\%) & $n$ & (\%) & $n$ & (\%) & \\
\hline$N(\%)$ & 223 & $(100)$ & 93 & $(41.7)$ & 126 & $(56.5)$ & - \\
\hline Age [year]: & & & & & & & 0.14 \\
\hline $18-30$ & 80 & $(37.0)$ & 36 & $(40.0)$ & 44 & (34.9) & \\
\hline $31-40$ & 75 & $(34.7)$ & 35 & $(38.9)$ & 40 & (31.8) & \\
\hline$>40$ & 61 & $(28.2)$ & 19 & $(21.1)$ & 42 & (33.3) & \\
\hline Age at onset of disease [years]: & & & & & & & $<0.001$ \\
\hline$<15$ & 56 & $(26.7)$ & 35 & $(39.3)$ & 21 & (17.4) & \\
\hline $15-25$ & 90 & $(42.9)$ & 37 & $(41.6)$ & 53 & $(43.8)$ & \\
\hline$>25$ & 64 & $(30.5)$ & 17 & $(19.1)$ & 47 & $(38.8)$ & \\
\hline Disease duration [year]: & & & & & & & 0.04 \\
\hline$<6$ & 53 & $(25.5)$ & 20 & $(23.0)$ & 33 & $(27.3)$ & \\
\hline $6-15$ & 75 & $(36.1)$ & 25 & $(28.7)$ & 50 & $(41.3)$ & \\
\hline$>15$ & 80 & $(38.5)$ & 42 & $(48.3)$ & 38 & (31.4) & \\
\hline Disease severity (sa-SPI-s): & & & & & & & 0.77 \\
\hline Mild $(<10)$ & 120 & $(57.1)$ & 54 & $(60.0)$ & 66 & $(55.0)$ & \\
\hline Moderate (10-20) & 55 & $(26.2)$ & 22 & $(24.4)$ & 33 & $(27.5)$ & \\
\hline Severe $(>20)$ & 35 & $(16.7)$ & 14 & $(15.6)$ & 21 & $(17.5)$ & \\
\hline Impact of psoriasis on daily life (sa-SPI-p): & & & & & & & 0.55 \\
\hline$<4$ & 64 & $(30.2)$ & 26 & $(28.3)$ & 38 & $(31.7)$ & \\
\hline $4-7$ & 67 & $(31.6)$ & 27 & $(29.4)$ & 40 & (33.3) & \\
\hline$>7$ & 81 & $(38.2)$ & 39 & $(42.4)$ & 42 & $(35.0)$ & \\
\hline Previous/ongoing depression diagnosis & 37 & $(17.1)$ & 12 & $(12.9)$ & 25 & $(20.2)$ & 0.16 \\
\hline Previous/current medication for depression & 11 & $(5.1)$ & 4 & $(4.3)$ & 7 & $(5.7)$ & 0.66 \\
\hline Previous/ongoing anxiety diagnosis & 76 & $(34.7)$ & 41 & $(44.1)$ & 35 & $(27.8)$ & 0.01 \\
\hline Previous/current medication for anxiety & 14 & $(6.5)$ & 5 & $(5.5)$ & 9 & $(7.1)$ & 0.63 \\
\hline Education level: & & & & & & & $<0.001$ \\
\hline Below secondary & 35 & $(16.1)$ & 10 & $(10.8)$ & 25 & $(20.2)$ & \\
\hline Secondary & 51 & $(23.5)$ & 12 & $(12.9)$ & 39 & (31.5) & \\
\hline Higher & 131 & $(60.4)$ & 71 & $(76.3)$ & 60 & $(48.4)$ & \\
\hline Marital status: & & & & & & & 0.003 \\
\hline Married & 128 & $(58.7)$ & 43 & $(46.2)$ & 85 & $(68.0)$ & \\
\hline Single & 85 & (39.0) & 46 & $(49.5)$ & 39 & (31.2) & \\
\hline Divorced & 5 & $(2.3)$ & 4 & (4.3) & 1 & $(0.8)$ & \\
\hline Country': & & & & & & & 0.72 \\
\hline Algeria & 64 & $(31.2)$ & 32 & $(38.1)$ & 32 & (26.5) & \\
\hline Iraq & 52 & $(25.4)$ & 20 & $(23.8)$ & 32 & (26.5) & \\
\hline Egypt & 25 & $(12.2)$ & 9 & $(10.7)$ & 16 & $(13.2)$ & \\
\hline Syria & 16 & (7.8) & 4 & (4.8) & 12 & (9.9) & \\
\hline Tunisia & 15 & $(7.3)$ & 6 & $(7.1)$ & 9 & (7.4) & \\
\hline Morocco & 14 & $(6.8)$ & 6 & $(7.1)$ & 8 & (6.6) & \\
\hline Jordan & 10 & $(4.9)$ & 4 & (4.8) & 6 & (5.0) & \\
\hline Other Arabic country & 9 & (4.4) & 3 & (3.6) & 6 & (5.0) & \\
\hline
\end{tabular}

Results are $n$ (\%). sa-SPI-s - self-assessed Simplified Psoriasis Index-severity scale, sa-SPI-p - self-assessed Simplified Psoriasis Index-psychosocial impact. ${ }^{\dagger}$ Country was missing for 18 patients. 


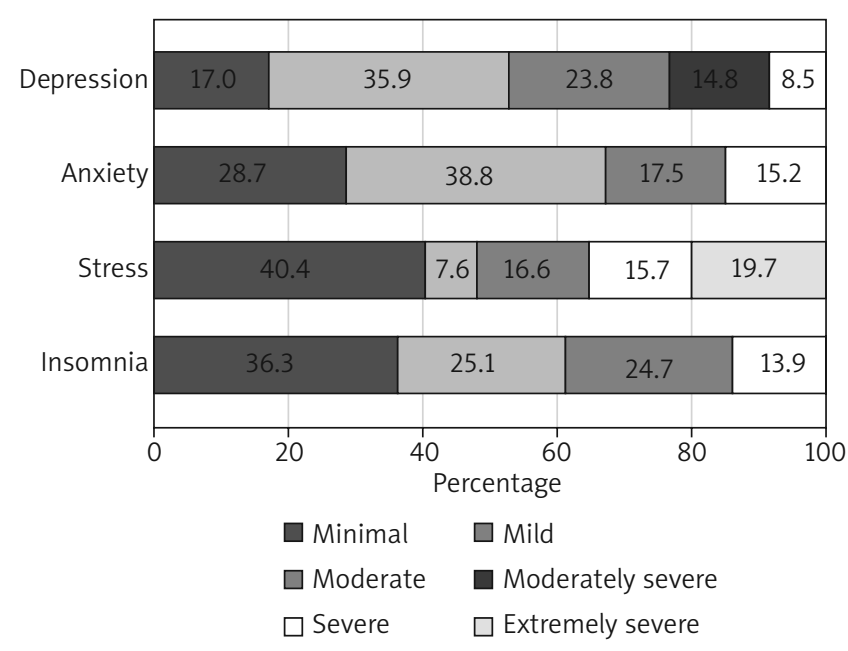

Figure 1. Distribution of depressive, anxiety, stress, and insomnia symptoms among the patients

\section{Anxiety symptoms}

Mild symptoms of anxiety were noted among patients with a median (IQR) GAD-7 score of 6 (4-11). The minimal anxiety level was reported by $28.7 \%$ of the patients, whereas $38.6 \%, 17.5 \%$, and $15.2 \%$ of the patients reported mild, moderate, and severe levels of anxiety, respectively (Figure 1). The prevalence of anxiety symptoms was $32.7 \%$ (95\% Cl: 26.5-38.9\%; Figure 2). In response to the questions, 181 (81.2\%) patients reported being nervous, anxious, or on the edge and 110 (49.3\%) patients reported being restless. In response to how difficult it was to perform daily life duties due to anxiety, 79 (35.6\%), 102 (46\%), 26 (11.7\%), and 15 (6.8\%) patients reported not difficult at all, somewhat difficult, very difficult, and extremely difficult, respectively.

Women had higher anxiety scores than men ( $p=$ 0.03). Patients with a higher impact of psoriasis on daily life had higher anxiety scores ( $p=0.001$; Table 2 ). Multivariate linear regression analysis for factors associated with the anxiety score revealed that for each unit in crease in impact of psoriasis on daily life (sa-SPI-p) there was 3.8 (95\% Cl: 1.8-5.7) units increase in anxiety score (GAD-7) for sa-SPI-p score > 7 (Table 3).

\section{Stress symptoms}

The median (IQR) stress subscale of DASS-21 score of the patients was 10 (5-14), indicating a moderate level of stress. Mild, moderate, severe, and extremely severe stress levels were noted in 7.6\%, 16.6\%, 15.7\%, and 19.7\% of the patients, respectively (Figure 1). The prevalence of stress symptoms was 59.6\% (95\% Cl: 53.1-66.0\%; Figure 2). In regard to the seven statements of the stress measure, 175 (78.5\%) patients reported getting agitated either sometimes, often, or almost always and 71 (31.8\%) patients reported feeling nervous almost always. Approxi-

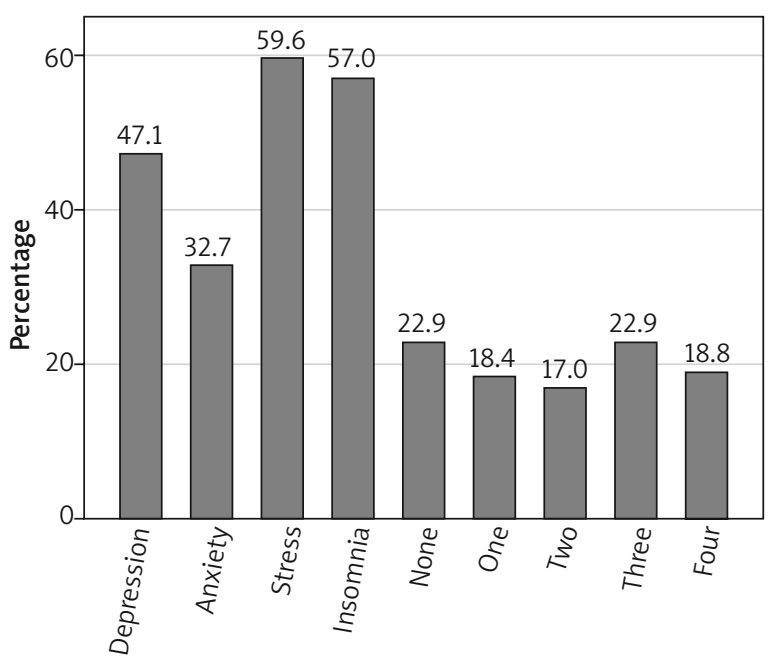

Figure 2. Prevalence of symptoms of depression, anxiety, stress, insomnia, none of the four conditions, one condition, two conditions, three conditions, and all four conditions among the patients

mately $30.9 \%$ of the patients reported that they never felt it hard to wind down over the past week.

Women had higher median (IQR) stress scores (12 $(6-16))$ than men $(8(4-14) ; p=0.02)$. Patients with severe psoriasis had the highest stress scores (13 (8-18)) compared with those with moderate $(10(5-14))$ and mild (9 (4-13)) disease $(p=0.04)$. Patients with a higher impact of psoriasis on their daily life had higher levels of stress ( $p=0.0001$; Table 4). Multivariate linear regression analysis for factors associated with the stress score revealed that the stress score (stress subscale of DASS-21) increased by 5.3 (95\% Cl: 3.1-7.4) units for each unit increase in the impact of psoriasis on daily life for (sa-SPI-p) score $>7$ (Table 3).

\section{Insomnia symptoms}

The median (IQR) ISI score of the patients was 12 (4-18), reflecting mild insomnia symptoms. In total, 81 (36.3\%), 56 (25.1\%), 55 (24.7\%), and 31 (13.9\%) patients reported minimal, mild, moderate, and severe insomnia symptoms, respectively (Figure 1). The prevalence of insomnia was 57\% (95\% Cl: 50.4-63.5\%; Figure 2). In regard to response to the seven items of the ISI, 57 (25.6\%) patients reported being very dissatisfied with their current sleep pattern and 55 (24.7\%) patients reported a very severe problem of waking up too early in the morning. However, 103 (46.2\%) patients reported that a sleep problem was not at all noticeable to others in terms of impaired QoL.

Patients with a higher impact of psoriasis on their daily life (sa-SPI-p) had higher levels of insomnia symptoms; the median (IQR) ISI score among patients with sa- 
Table 2. Prevalence and scores of depressive and anxiety symptoms among the patients stratified by sociodemographics and clinical characteristics

\begin{tabular}{|c|c|c|c|c|c|c|c|c|c|c|c|c|}
\hline \multirow{4}{*}{$\begin{array}{l}\text { Variables } \\
\text { Total } 223 \text { patients }\end{array}$} & \multicolumn{6}{|c|}{ Depressive symptoms (PHQ-9 score) } & \multicolumn{6}{|c|}{ Anxiety symptoms (GAD-7 score) } \\
\hline & \multicolumn{2}{|c|}{ Prevalence } & \multirow{3}{*}{$\begin{array}{c}\text { Median } \\
9.0\end{array}$} & \multirow{2}{*}{\multicolumn{2}{|c|}{ IQR }} & \multirow{3}{*}{$\begin{array}{c}P \text {-value } \\
-\end{array}$} & \multicolumn{2}{|c|}{ Prevalence } & \multirow{3}{*}{$\begin{array}{c}\text { Median } \\
6.0\end{array}$} & \multirow{2}{*}{\multicolumn{2}{|c|}{ IQR }} & \multirow{2}{*}{$P$-value ${ }^{\dagger}$} \\
\hline & \multirow{2}{*}{$\begin{array}{r}n \\
105\end{array}$} & \multirow{2}{*}{$\begin{array}{c}\% \\
47.1\end{array}$} & & & & & \multirow{2}{*}{$\begin{array}{c}n \\
73\end{array}$} & \multirow{2}{*}{$\begin{array}{l}\% \\
32.7\end{array}$} & & & & \\
\hline & & & & 6.0 & 14.0 & & & & & 4.0 & 11.0 & - \\
\hline Age [year]: & & & & & & 0.46 & & & & & & 0.66 \\
\hline $18-30$ & 40 & 50.0 & 9.5 & 5.5 & 13.0 & & 29 & 36.3 & 7.0 & 4.0 & 12.5 & \\
\hline $31-40$ & 38 & 50.7 & 10.0 & 6.0 & 16.0 & & 26 & 34.7 & 6.0 & 4.0 & 12.0 & \\
\hline$>40$ & 25 & 41.0 & 9.0 & 6.0 & 12.0 & & 16 & 26.2 & 6.0 & 4.0 & 10.0 & \\
\hline Gender: & & & & & & 0.02 & & & & & & 0.03 \\
\hline Males & 55 & 43.7 & 9.0 & 6.0 & 12.0 & & 35 & 27.8 & 6.0 & 3.0 & 10.0 & \\
\hline Females & 50 & 53.8 & 11.0 & 7.0 & 16.0 & & 37 & 39.8 & 7.0 & 5.0 & 12.0 & \\
\hline \multicolumn{2}{|c|}{ Psoriasis duration [year]: } & & & & & 0.07 & & & & & & \\
\hline$<6$ & 24 & 45.3 & 9.0 & 6.0 & 13.0 & & 20 & 37.7 & 8.0 & 4.0 & 12.0 & 0.43 \\
\hline $6-15$ & 29 & 38.7 & 8.0 & 5.0 & 12.0 & & 21 & 28.0 & 6.0 & 3.0 & 10.0 & \\
\hline$>15$ & 46 & 57.5 & 11.0 & 6.0 & 16.0 & & 27 & 33.8 & 6.5 & 4.5 & 12.0 & \\
\hline \multicolumn{2}{|c|}{ Psoriasis severity (sa-SPI-s): } & & & & & 0.0004 & & & & & & 0.16 \\
\hline Mild $(<10)$ & 46 & 37.7 & 8.0 & 5.0 & 12.0 & & 37 & 30.3 & 6.0 & 3.0 & 11.0 & \\
\hline Moderate (10-20) & 27 & 49.1 & 9.0 & 6.0 & 14.0 & & 16 & 29.1 & 7.0 & 5.0 & 10.0 & \\
\hline Severe $(>20)$ & 26 & 74.3 & 13.0 & 9.0 & 16.0 & & 16 & 45.7 & 8.0 & 5.0 & 15.0 & \\
\hline \multicolumn{4}{|c|}{ Impact of psoriasis on daily life (sa-SPI-p): } & & & 0.0001 & & & & & & 0.001 \\
\hline$<4$ & 18 & 28.1 & 7.0 & 4.0 & 10.5 & & 10 & 15.6 & 5.5 & 2.0 & 8.0 & \\
\hline $4-7$ & 25 & 36.2 & 8.0 & 6.0 & 12.0 & & 26 & 37.7 & 6.0 & 4.0 & 10.0 & \\
\hline$>7$ & 58 & 69.9 & 12.0 & 8.0 & 18.0 & & 35 & 42.2 & 8.0 & 5.0 & 15.0 & \\
\hline Education level: & & & & & & 0.81 & & & & & & 0.34 \\
\hline Primary & 16 & 45.7 & 9.0 & 6.0 & 16.0 & & 12 & 34.3 & 7.0 & 5.0 & 11.0 & \\
\hline Secondary & 29 & 56.9 & 10.0 & 7.0 & 12.0 & & 16 & 31.4 & 7.0 & 5.0 & 12.0 & \\
\hline Higher & 59 & 45.0 & 9.0 & 5.0 & 15.0 & & 43 & 32.8 & 6.0 & 4.0 & 11.0 & \\
\hline
\end{tabular}

PHQ-9-Patient Health Questionnaire, GAD-7 - Generalized Anxiety Disorder 7-item scale, sa-SPI-s-self-assessed Simplified Psoriasis Index-severity scale, sa-SPI-p - self-assessed Simplified Psoriasis Index-psychosocial impact. ${ }^{\text {T}}$ The Kruskal-Wallis test by ranks or Wilcoxon signed-rank test.

SPI-p scores > 7 was 17 (8-22), compared with 11 (5-16) among patients with sa-SPI-p scores of 4-7 and 7 (2-13) among patients with sa-SPI-p scores $<4$ ( $p=0.0001$; Table 4). Multivariate linear regression analysis for factors associated with ISI score revealed that for each unit increase in impact of psoriasis on daily life (sa-SPI-p) there was 3.2 (95\% CI: 0.4-6.0) units increase in ISI score for sa-SPI-p score ranging from 4 to 7 and $6.5(95 \% \mathrm{Cl}$ : 3.7-9.4) for sa-SPI-p score > 7 (Table 3).

\section{Discussion}

In this study, the levels and prevalence of depressive, anxiety, stress, and insomnia symptoms were assessed among Arabic patients with psoriasis using patientreported instruments, namely the PHQ-9, GAD-7, stress subscale of the DASS-21, and ISI. The patients showed mild symptoms of depression, anxiety, and insomnia and a moderate level of stress. The prevalence of depressive, anxiety, stress, and insomnia symptoms were $47.1 \%$, $32.7 \%, 59.6 \%$, and $57 \%$, respectively.

In the current study, the prevalence of depressive and anxiety symptoms was higher than the pooled value reported in a meta-analysis on depressive symptoms in patients with psoriasis, which reported a prevalence of $28 \%$ by assessing patient questionnaires [3]. In addition, the prevalence of depressive and anxiety symptoms were higher than those noted among 208 Chinese patients with psoriasis (depressive symptoms: $13.9 \%$ and anxiety symptoms: 10.6\%) [27], comparable to that among 50 patients with psoriasis vulgaris from Turkey (depressive symptoms: 58\%) [28], and lower than those among 90 Indian patients with psoriasis (depressive symptoms: $78.9 \%$ and anxiety symptoms: 76.7\%) [29]. The median (IQR) PHQ-9 score in the current study was 9 (6-14), which is higher than that reported in a study on 
Table 3. Results of multivariate linear regression analysis of factors associated with depressive, anxiety, stress, and insomnia symptoms

\begin{tabular}{|c|c|c|c|c|c|c|c|c|c|c|c|c|}
\hline \multirow[t]{2}{*}{ Variables } & \multicolumn{3}{|c|}{$\begin{array}{l}\text { Depression } \\
\text { (PHQ-9 score) }\end{array}$} & \multicolumn{3}{|c|}{$\begin{array}{c}\text { Anxiety } \\
\text { (GAD-7 score) }\end{array}$} & \multicolumn{3}{|c|}{$\begin{array}{c}\text { Stress } \\
\text { (DASS-21 stress score) }\end{array}$} & \multicolumn{3}{|c|}{$\begin{array}{l}\text { Insomnia } \\
\text { (ISI score) }\end{array}$} \\
\hline & $\beta$ Coef. & $95 \% \mathrm{Cl}$ & $P$-value & $\beta$ Coef. & $95 \% \mathrm{Cl}$ & $P$-value & $\beta$ Coef. & $95 \% \mathrm{Cl}$ & $P$-value & $\beta$ Coef. & $95 \% \mathrm{Cl}$ & $P$-value \\
\hline \multicolumn{13}{|l|}{ Age [year]: } \\
\hline $18-30$ & Reference & & & & & & & & & & & \\
\hline $31-40$ & 1.1 & $-0.9,3.2$ & 0.27 & -0.5 & $-2.4,1.4$ & 0.60 & 1.3 & $-0.8,3.4$ & 0.23 & 2.3 & $-0.5,5.1$ & 0.11 \\
\hline$>40$ & 0.5 & $-1.7,2.7$ & 0.65 & -0.6 & $-2.6,1.5$ & 0.58 & 1.6 & $-0.7,3.8$ & 0.17 & 1.2 & $-1.8,4.2$ & 0.43 \\
\hline \multicolumn{13}{|l|}{ Gender: } \\
\hline Males & Reference & & & & & & & & & & & \\
\hline Females & 1.3 & $-0.4,3.1$ & 0.14 & 1.4 & $-0.3,3.0$ & 0.10 & 1.7 & $-0.1,3.5$ & 0.06 & 0.8 & $-1.6,3.2$ & 0.53 \\
\hline \multicolumn{13}{|c|}{ Psoriasis duration [year]: } \\
\hline$<6$ & Reference & & & & & & & & & & & \\
\hline $6-15$ & -1.7 & $-3.8,0.3$ & 0.10 & -0.6 & $-2.6,1.3$ & 0.51 & -0.6 & $-2.7,1.5$ & 0.55 & -1.1 & $-4.0,1.7$ & 0.42 \\
\hline$>15$ & 0.1 & $-2.2,2.3$ & 0.96 & -0.2 & $-2.3,1.9$ & 0.84 & -1.0 & $-3.3,1.3$ & 0.39 & -0.9 & $-3.9,2.2$ & 0.58 \\
\hline \multicolumn{13}{|c|}{ Psoriasis severity (sa-SPI-s): } \\
\hline Mild $(<10)$ & Reference & & & & & & & & & & & \\
\hline Moderate (10-20) & 0.4 & $-1.5,2.3$ & 0.66 & -0.1 & $-1.9,1.7$ & 0.93 & -0.4 & $-2.3,1.6$ & 0.72 & -0.5 & $-3.1,2.1$ & 0.70 \\
\hline Severe $(>20)$ & 1.3 & $-1.2,3.7$ & 0.32 & 0.9 & $-1.4,3.2$ & 0.45 & 0.7 & $-1.8,3.3$ & 0.59 & 0.0 & $-3.4,3.4$ & 0.99 \\
\hline \multicolumn{13}{|c|}{ Impact of psoriasis on daily life (sa-SPI-p): } \\
\hline$<4$ & Reference & & & & & & & & & & & \\
\hline $4-7$ & 2.8 & $0.8,4.9$ & 0.01 & 1.8 & $-0.1,3.7$ & 0.07 & 1.9 & $-0.2,4.0$ & 0.08 & 3.2 & $0.4,6.0$ & 0.03 \\
\hline$>7$ & 5.7 & $3.6,7.8$ & $<0.001$ & 3.8 & $1.8,5.7$ & $<0.001$ & 5.3 & $3.1,7.4$ & $<0.001$ & 6.5 & $3.7,9.4$ & $<0.001$ \\
\hline \multicolumn{13}{|l|}{ Education level: } \\
\hline Primary & Reference & & & & & & & & & & & \\
\hline Secondary & -0.8 & $-3.5-1.9$ & 0.55 & 0.2 & $-2.3,2.7$ & 0.85 & -0.2 & $-2.9,2.6$ & 0.91 & 1.8 & $-1.9,5.5$ & 0.33 \\
\hline Higher & -0.9 & $-3.2-1.5$ & 0.48 & -1.2 & $-3.4,1.0$ & 0.28 & -1.9 & $-4.3,0.6$ & 0.13 & 0.8 & $-2.4,4.0$ & 0.63 \\
\hline
\end{tabular}

PHQ-9 - Patient Health Questionnaire, GAD-7 - Generalized Anxiety Disorder 7-item scale, DASS-21 - Depression Anxiety Stress Scale, ISI - Insomnia Severity Index, sa-SPI-s - self-assessed Simplified Psoriasis Index-severity scale, sa-SPI-p - self-assessed Simplified Psoriasis Index-psychosocial impact.

351 American patients with psoriasis (mean \pm SD: PHQ-9 score: $4.54 \pm 5.7$ ) [30]. This heterogeneity in reported values may be explained by different ethnicity of the patients, different instruments used for screening, and different medical care provided to each population. The selection bias may explain, to an extent, the high prevalence of psychological symptoms because patients with psychological distress may be more frequently visiting the clinics to seek more help, which increases their probability to participate in a study or they may be more willing to fill in questionnaires regarding depressive, anxiety, or stress symptoms.

In the current study, the prevalence of stress symptoms was $59.6 \%$. In a cross-sectional study of 60 psoriasis patients from Brazil, the prevalence of stress was reported to be $85 \%$ [31]. A systematic review indicated that the psoriasis-related overall stress rates in epidemiological studies ranged from $27 \%$ to $71 \%$ [5]. Another systematic review and meta-analysis, including 39 stud- ies, indicated that $46 \%$ of the patients believed that their psoriasis was stress reactive and 54\% linked preceding stressful events to psoriasis worsening [32].

The median (IQR) ISI score representing mild insomnia symptoms in the current study was 12 (4-18), which was lower than that reported (19.7) in a study of $60 \mathrm{pa}-$ tients with plaque psoriasis from Turkey [33]. The insomnia in psoriasis patients may be related to pruritus and pain caused by the disease itself or to other comorbidities such as depression or obesity [34].

Although the current study did not assess the prevalence and level of suicidality using a specific instrument, the ninth question of the PHQ-9 provided an idea regarding suicidal ideation, which was prevalent in 18.4\% (95\% Cl: $13.3-23.5 \%)$ of the patients. In a cross-sectional study involving 607 psoriasis patients from the UK, suicidal ideation was noted among 3.5\% (95\% Cl: 2-4.9\%) of the patients as assessed using the ninth question of the PHQ 9 [35]. The higher prevalence of suicidal ideation 
Table 4. Prevalence and scores of stress and insomnia symptoms among the patients stratified by socio-demographics and clinical characteristics

\begin{tabular}{|c|c|c|c|c|c|c|c|c|c|c|c|c|}
\hline \multirow{4}{*}{$\begin{array}{l}\text { Variables } \\
\text { Total } 223 \text { patients }\end{array}$} & \multicolumn{6}{|c|}{ Stress symptoms (DASS-21 stress score) } & \multicolumn{6}{|c|}{ Insomnia symptoms (ISI score) } \\
\hline & \multicolumn{2}{|c|}{ Prevalence } & \multirow{3}{*}{$\begin{array}{c}\text { Median } \\
10.0\end{array}$} & \multirow{2}{*}{\multicolumn{2}{|c|}{ IQR }} & \multirow{3}{*}{$\begin{array}{c}P \text {-value } \\
-\end{array}$} & \multicolumn{2}{|c|}{ Prevalence } & \multirow{3}{*}{$\begin{array}{c}\text { Median } \\
12.0\end{array}$} & \multirow{2}{*}{\multicolumn{2}{|c|}{ IQR }} & \multirow[t]{2}{*}{$P$-value ${ }^{\dagger}$} \\
\hline & & \multirow{2}{*}{$\begin{array}{c}\% \\
59.6\end{array}$} & & & & & \multirow{2}{*}{$\frac{n}{127}$} & \multirow{2}{*}{$\begin{array}{c}\% \\
57.0\end{array}$} & & & & \\
\hline & & & & 5.0 & 14.0 & & & & & 4.0 & 18.0 & - \\
\hline Age [year]: & & & & & & 0.87 & & & & & & 0.30 \\
\hline $18-30$ & 47 & 58.8 & 10.0 & 5.0 & 14.0 & & 40 & 50.0 & 9.5 & 2.0 & 18.5 & \\
\hline $31-40$ & 47 & 62.7 & 10.0 & 4.0 & 17.0 & & 47 & 62.7 & 13.0 & 7.0 & 19.0 & \\
\hline$>40$ & 34 & 55.7 & 9.0 & 5.0 & 13.0 & & 36 & 59.0 & 12.0 & 4.0 & 17.0 & \\
\hline Gender: & & & & & & 0.02 & & & & & & 0.30 \\
\hline Males & 67 & 53.2 & 8.0 & 4.0 & 14.0 & & 70 & 55.6 & 11.0 & 3.0 & 17.0 & \\
\hline Females & 64 & 68.8 & 12.0 & 6.0 & 16.0 & & 55 & 59.1 & 13.0 & 6.0 & 20.0 & \\
\hline Psoriasis duration [ye & & & & & & 0.70 & & & & & & 0.55 \\
\hline$<6$ & 30 & 56.6 & 9.0 & 6.0 & 16.0 & & 32 & 60.4 & 12.0 & 4.0 & 20.0 & \\
\hline $6-15$ & 41 & 54.7 & 9.0 & 5.0 & 14.0 & & 41 & 54.7 & 11.0 & 3.0 & 16.0 & \\
\hline$>15$ & 53 & 66.3 & 10.5 & 4.0 & 15.0 & & 45 & 56.3 & 12.0 & 4.5 & 18.0 & \\
\hline Psoriasis severity (sa- & & & & & & 0.04 & & & & & & 0.06 \\
\hline Mild $(<10)$ & 67 & 54.9 & 9.0 & 4.0 & 13.0 & & 65 & 53.3 & 10.0 & 3.0 & 17.0 & \\
\hline Moderate (10-20) & 33 & 60.0 & 10.0 & 5.0 & 14.0 & & 32 & 58.2 & 12.0 & 7.0 & 16.0 & \\
\hline Severe $(>20)$ & 27 & 77.1 & 13.0 & 8.0 & 18.0 & & 24 & 68.6 & 17.0 & 5.0 & 22.0 & \\
\hline Impact of psoriasis or & ily life & $-\mathrm{SPI}-\mathrm{p})$ & & & & 0.0001 & & & & & & 0.0001 \\
\hline$<4$ & 25 & 39.1 & 7.0 & 3.0 & 11.0 & & 27 & 42.2 & 7.0 & 2.0 & 13.0 & \\
\hline $4-7$ & 40 & 58.0 & 9.0 & 4.0 & 14.0 & & 39 & 56.5 & 11.0 & 5.0 & 16.0 & \\
\hline$>7$ & 65 & 78.3 & 13.0 & 8.0 & 18.0 & & 58 & 69.9 & 17.0 & 8.0 & 22.0 & \\
\hline Education level: & & & & & & 0.43 & & & & & & 0.43 \\
\hline Primary & 22 & 62.9 & 11.0 & 5.0 & 15.0 & & 15 & 57.1 & 8.0 & 2.0 & 17.0 & \\
\hline Secondary & 33 & 64.7 & 11.0 & 5.0 & 16.0 & & 33 & 35.3 & 12.0 & 6.0 & 18.0 & \\
\hline Higher & 75 & 57.3 & 9.0 & 5.0 & 14.0 & & 76 & 42.0 & 12.0 & 5.0 & 18.0 & \\
\hline
\end{tabular}

DASS-21 - Depression Anxiety Stress Scale, ISI - Insomnia Severity Index, sa-SPI-s - self-assessed Simplified Psoriasis Index-severity scale, sa-SPI-P-self-assessed Simplified Psoriasis Index-psychosocial impact. ${ }^{+}$The Kruskal-Wallis test by ranks or Wilcoxon signed-rank test.

in the current study suggests that studies on suicidal ideation using a specific instrument and a control group are warranted among the Arabic patients with psoriasis in the future.

Socio-demographic variables were found to be not associated with psychological disorders in the current study because the prevalence of psychological disorders did not change with age and education level, which is in agreement with the literature $[29,36]$. Depressive, anxiety, and stress symptoms were found to be at higher levels and more prevalent among women, which is in agreement with the findings of a study involving 2579 dermatological patients from Italy [36]. However, the gender effect disappeared after adjusting for other variables in the multivariate analysis. This was not surprising because women are more likely to show depressive and anxiety symptoms in general [37] (i.e., not specifically due to psoriasis). Disease duration did not affect the fre- quency or magnitude of psychological symptoms, which is in agreement with the findings of previous studies [28]. According to the multivariate linear regression analysis in the current study, the impact of psoriasis on daily life was the only significant predictor of higher levels of depressive, anxiety, stress, and insomnia symptoms. Similar findings were noted in the literature, psoriasis with comorbid anxiety/depression was significantly associated with worse QoL $[29,36]$.

According to our knowledge, this is the first study to assess psychological distress and insomnia in Arabic patients with psoriasis. Because this study was not limited to a hospital, medical centre, or country, the generalizability of the results to the entire Arab community is assured. However, the absence of a control group may limit the generalizability of the results to all psoriasis patients.

Although the cross-sectional design was convenient, cost effective, rapid, and easy to conduct, it limited the 
evaluation of the causal ordering of variable relationships. The online survey method provided sufficient and suitable time to the patients for completing the survey and allowed collection of responses from 11 Arab countries.

Selection bias may be a concern in the current study because probably psoriasis patients with no symptoms of psychological distress probably did not relate themselves to the study and hence, chose not to participate. However, when administering the survey, it was highlighted that all psoriasis patients should participate regardless of having psychological issues.

\section{Conclusions}

The prevalence of depressive, anxiety, stress, and insomnia symptoms among Arabic patients with psoriasis was high. Patients with a higher impact of psoriasis on their daily life were more likely to have psychological distress. Clinical interventions, patient counselling, screening for psychiatric comorbidities, and consideration of psychotherapy should be implemented in this patient group to reduce the burden of psychological distress and subsequent disease complications and QoL impairment.

\section{Acknowledgments}

The author would like to thank all study participants for completing the survey.

\section{Conflict of interest}

The author declares no conflict of interest.

\section{References}

1. Rapp SR, Feldman SR, Exum ML, et al. Psoriasis causes as much disability as other major medical diseases. J Am Acad Dermatol 1999; 41: 401-7.

2. Sampogna F, Tabolli S, Mastroeni S, et al. Quality of life impairment and psychological distress in elderly patients with psoriasis. Dermatology 2007; 215: 341-7.

3. Dowlatshahi EA, Wakkee M, Arends LR, Nijsten T. The prevalence and odds of depressive symptoms and clinical depression in psoriasis patients: a systematic review and metaanalysis. J Invest Dermatol 2014; 134: 1542-51.

4. Elsayed M, Connor CJ. Beneath the skin: the relationship between psychological distress and the immune system in patients with psoriasis. EMJ Dermatol 2018; 6: 108-17.

5. Stewart TJ, Tong W, Whitfeld MJ. The associations between psychological stress and psoriasis: a systematic review. Int J Dermatol 2018; 57: 1275-82.

6. Fried RG. Nonpharmacologic treatments in psychodermatology. Dermatol Clin 2002; 20: 177-85.

7. Kim SJ, Park MY, Pak K, et al. Improvement of depressive symptoms in patients with moderate-to-severe psoriasis treated with ustekinumab: an open label trial validated using beck depression inventory, Hamilton depression rating scale measures and (18)fluorodeoxyglucose (FDG) positron. J Dermatolog Treat 2018; 29: 761-8.
8. Langley RG, Feldman SR, Han C, et al. Ustekinumab significantly improves symptoms of anxiety, depression, and skin-related quality of life in patients with moderateto-severe psoriasis: results from a randomized, doubleblind, placebo-controlled phase III trial. J Am Acad Dermatol 2010; 63: 457-65.

9. Gordon KB, Armstrong AW, Han C, et al. Anxiety and depression in patients with moderate-to-severe psoriasis and comparison of change from baseline after treatment with guselkumab vs. adalimumab: results from the Phase 3 VOYAGE 2 study. J Eur Acad Dermatol Venereol 2018; 32: 1940-9.

10. Menter A, Augustin M, Signorovitch J, et al. The effect of adalimumab on reducing depression symptoms in patients with moderate to severe psoriasis: a randomized clinical trial. J Am Acad Dermatol 2010; 62: 812-8.

11. AlShahwan MA. The prevalence of anxiety and depression in Arab dermatology patients. J Cutan Med Surg 2015; 19: 297-303.

12. Ahmed AE, Al-Dahmash AM, Al-Boqami QT, Al-Tebainawi YF. Depression, anxiety and stress among Saudi Arabian dermatology patients: cross-sectional study. Sultan Qaboos Univ Med J 2016; 16: 217-23.

13. Al Alawi M, Al Sinawi H, Al Qasabi AM, et al. Prevalence and predictors of depressive symptoms among attendees of a tertiary care dermatology clinic in Muscat, Oman. Int J Dermatol 2018; 57: 284-90.

14. Mleeh NT, Alshamrani HM, Basyouni RN, et al. Prevalence and predictors of depression among dermatology clinic patients in a teaching hospital, Jeddah, Saudi Arabia. J Fam Med Prim Care 2019; 8: 2496-501.

15. Chularojanamontri L, Griffiths CEM, Chalmers RJG. The Simplified Psoriasis Index (SPI): a practical tool for assessing psoriasis. J Invest Dermatol 2013; 133: 1956-62.

16. Daniel WW, Cross CL. Biostatistics: a foundation for analysis in the health sciences. Wiley 2018.

17. Pourhoseingholi MA, Vahedi M, Rahimzadeh M. Sample size calculation in medical studies. Gastroenterol Hepatol Bed Bench 2013; 6: 14-7.

18. Kroenke K, Spitzer RL, Williams JB. The PHQ-9: validity of a brief depression severity measure. J Gen Intern Med 2001; 16: 606-13.

19. AlHadi AN, AlAteeq DA, Al-Sharif E, et al. An Arabic translation, reliability, and validation of Patient Health Questionnaire in a Saudi sample. Ann Gen Psychiatry 2017; 16: 32.

20. Spitzer RL, Kroenke K, Williams JBW, Löwe B. A brief measure for assessing generalized anxiety disorder: the GAD-7. Arch Intern Med 2006; 166: 1092-7.

21. Henry JD, Crawford JR. The short-form version of the Depression Anxiety Stress Scales (DASS-21): construct validity and normative data in a large non-clinical sample. Br J Clin Psychol 2005; 44: 227-39.

22. Lovibond PF, Lovibond SH. The structure of negative emotional states: comparison of the Depression Anxiety Stress Scales (DASS) with the Beck Depression and Anxiety Inventories. Behav Res Ther 1995; 33: 335-43.

23. Ali AM, Ahmed A, Sharaf A, et al. The Arabic Version of The Depression Anxiety Stress Scale-21: cumulative scaling and discriminant-validation testing. Asian J Psychiatry 2017; 30: 56-8.

24. Bastien $\mathrm{CH}$, Vallières A, Morin CM. Validation of the Insomnia Severity Index as an outcome measure for insomnia research. Sleep Med 2001; 2: 297-307. 
25. Morin CM, Belleville G, Bélanger L, Ivers H. The Insomnia Severity Index: psychometric indicators to detect insomnia cases and evaluate treatment response. Sleep 2011; 34: 601-8.

26. Suleiman $\mathrm{KH}$, Yates BC. Translating the insomnia severity index into Arabic. J Nurs Scholarsh 2011; 43: 49-53.

27. Tian Z, Huang Y, Yue T, et al. A Chinese cross-sectional study on depression and anxiety symptoms in patients with psoriasis vulgaris. Psychol Health Med 2019; 24: 269-80.

28. Akay A, Pekcanlar A, Bozdag KE, et al. Assessment of depression in subjects with psoriasis vulgaris and lichen planus. J Eur Acad Dermatol Venereol 2002; 16: 347-52.

29. Lakshmy S, Balasundaram S, Sarkar S, et al. A cross-sectional study of prevalence and implications of depression and anxiety in psoriasis. Indian J Psychol Med 2015; 37: 434-40.

30. Cohen BE, Martires KJ, Ho RS. Psoriasis and the risk of depression in the US population: National Health and Nutrition Examination Survey 2009-2012. JAMA Dermatol 2016; 152: 73-9.

31. Leovigildo ÉS, David RAR, Mendes AS. Stress level of people with psoriasis at a public hospital. An Bras Dermatol 2016; 91: 446-54.

32. Snast I, Reiter O, Atzmony L, et al. Psychological stress and psoriasis: a systematic review and meta-analysis. Br J Dermatol 2018; 178: 1044-55.

33. Saçmacı H, Gürel G. Sleep disorders in patients with psoriasis: a cross-sectional study using non-polysomnographical methods. Sleep Breath 2019; 23: 893-8.

34. Gupta MA, Simpson FC, Gupta AK. Psoriasis and sleep disorders: a systematic review. Sleep Med Rev 2016; 29: 63-75.

35. Lamb RC, Matcham F, Turner MA, et al. Screening for anxiety and depression in people with psoriasis: a cross-sectional study in a tertiary referral setting. Br I Dermatol 2017; 176: 1028-34.

36. Picardi A, Abeni D, Melchi CF, et al. Psychiatric morbidity in dermatological outpatients: an issue to be recognized. Br J Dermatol 2000; 143: 983-91.

37. Horwath E, Weissman MM. Epidemiology of depression and anxiety disorders. In: Textbook in Psychiatric Epidemiology. Tsuang MT, Tohen M, Zahner GEP (eds). Wiley, New York 1995; 317-44. 